\title{
Aktuelle Beiträge zur tanzthera- peutischen Arbeit mit Flüchtlingen und MigrantInnen im Kontext von Gesellschaft und Menschenhandel
}

\author{
Iris Bräuninger
}

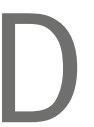

ieser Betrag stellt verschiedene Rahmenbedingungen und zwei Studien (eine Interventions- und eine Fallstudie) für die tanz-, bewegungstherapeutische Arbeiten mit Flüchtlingen und MigrantInnen im sozialen Kontext vor und berichtet über ein Programm zur Bekämpfung des Menschenhandels mit tanz-, bewegungstherapeutischem Ansatz.

\section{Reduktion von Trauma, Stress und} Angst bei syrischen Flüchtlingskindern durch Tanz / Bewegungstherapie

Als Teil eines größeren gemeindebasierten Programms für syrische Flüchtlingskinder gegen Trauma, Stress und Angst wurde über 12 Wochen Tanz-, Bewegungstherapie (TT) als Gruppenintervention über 90 Minuten angeboten (Grasser et al. 2019). Um die Teilnahme der Kinder zu fördern und ihre Trennungsangst zu reduzieren (Prävalenz 74,9\%), fand für die Mütter parallel zur TT achtsamkeitsbasiertes Yoga auf Arabisch statt, und ein kostenloser Busshuttle wurde für den Transport zu den Stunden bereitgestellt. Es nahmen 25 Kinder zwischen 7 und 14 Jahren teil, wovon 20 alle Therapiestunden besuchten. Der Datenerfassung und -verarbeitung stimmten $16 \mathrm{zu}(80 \%)$ (je 8 Mädchen und Jungen, Durchschnittsalter 10 Jahre), vier Elternteile nahmen am Yoga teil. Das TT-Programm startete mit der gesamten Gruppe im Kreis, gefolgt von Führen und Folgen und Spiegeln von Bewegung, Atem-, Dehnungsund Spannungsübungen, Bewegungsspielen, Ausdruck durch Bilder etc. und endete mit einem Abschlusskreis. Die Kinder beurteilten im Prätest und Posttest ihre posttraumatischen Stresssymptome anhand eines 33-Punkte-Fragebogens (UCLA PTSD) und ihre Angstsymptome anhand eines 41-Punkte-Fragebogens (SCARED). Die Daten wurden unter Verwendung des gepaarten Proben-t-Tests analysiert. Der Prätest-Posttest-Vergleich zeigte eine Reduktion von posttraumatischem Stress, Angstzuständen, allgemeinen Angstzuständen, Trennungsangst und Paniksymptomen.

\section{Kolkata-Sanved-Programm zur Bekämpfung von Menschenhandel}

Die Organisation Kolkata Sanved (KS) ist ein gemeindebasiertes, nicht-klinisches NGO-Programm, das 2004 von Sohini Chakraborty zur Bekämpfung von Menschenhandel in Kalkutta begründet wurde. In der Fachzeitschrift Child \& Abuse (Chakraborty 2020) stellt sie in ihrem 
Artikel für das Themenheft „Kinderhandel und Gesundheit“ eine spezielle Form der Tanzbewegungstherapie vor. „Sampoornata“ wurde als Form der Traumatherapie für Überlebende von sexueller Gewalt, Missbrauch, Ausbeutung und sexuellem Menschenhandel als Gruppenintervention entwickelt, „um Heilung und Wohlbefinden durch Integration in den sozialen Rahmen des Einzelnen und durch Förderung eines umfassenden Wohlbefindens zu fördern“ (S.1, Übersetzung durch I. Bräuninger). Am Programm können weibliche Überlebende teilnehmen, die als Kind oder Erwachsene Übergriffen oder Missbrauch ausgesetzt waren. KS arbeitet mit über 50 Organisationen und Regierungen in Indien und Südasien zusammen und bringt das Programm in gemeindebasierten Settings wie Pflegeheimen, Gesundheitseinrichtungen und Schulen ein.

KS bildet Heilerinnen und Tanztherapeutinnen aus, welche selbst Menschenhandel überlebt haben. $70 \%$ von ihnen stammen aus marginalisierten Randgruppen. Das Ziel der TT-Ausbildung ist, diese Frauen zu bestärken (empowerment), sie durch tägliche Bewegungskreise und monatliche Psychotherapiegruppen in ihrer Selbstfürsorge (self care) zu unterstützen, Intervisionsgruppen zu etablieren und durch sie als Multiplikatorinnen weitere Randgruppen zu erreichen. Während der Rehabilitation scheint für den Heilungsprozess die fortlaufende Anpassung an die Bedürfnisse der Überlebenden mit klaren Strukturen wichtig zu sein, was für die Tanztherapie bedeutet, dass lokaler Tanz, Musik und künstlerische Elemente in den Prozess integriert werden. Der Sampoornata-Prozess enthält folgende Elemente:

1. Bedürfnis-Assessment

2. Entwicklung eines Plans zur Implementierung

3. TT-Stunde (welche die nachfolgend beschriebenen acht Hauptaktivitäten integriert)
4. Dokumentation des Prozesses

5. Evaluation.

Jede Tanzbewegungstherapie-Stunde basiert auf acht Hauptaktivitäten:

1. Eröffnungsrituale

2. Warm-Up

3. Bedürfnisorientierte therapeutische Aktivitäten

4. Entspannung

5. Heilende Berührung

6. Abschließendes Ritual

7. Gruppendiskussionen als Feedback und Reflexion

8. Debriefing

Basierend auf ihrer langjährigen Erfahrung weist Chakraborty auf die große Bedeutung von Management- und Finanzierungsfragen hin, welche ausschlaggebend für die Akzeptanz und den Erfolg dieses psychosozialen, kreativen, nicht-klinischen Programms zur Bekämpfung des Menschenhandels durch Tanztherapie war und ist.

\section{Tanztherapeutische Arbeit mit}

Flüchtlingen und MigrantInnen, transkulturelle Haltung und

\section{Einbeziehung kultureller Elemente} und Empfehlungen

Zwei Übersichtspapiere und eine Fallvorstellung setzen sich mit der tanz-, bewegungstherapeutischen Arbeit mit Flüchtlingen auseinander: Aranda, Hills de Zárate und Panhofer (2020) machen Vorschläge für die transkulturelle tanz-, bewegungstherapeutische Arbeit mit ZwangsmigrantInnen und untersuchen Faktoren, welche die therapeutische Beziehung im Hinblick auf potenzielle blinde Flecken oder Hindernisse für das Verständnis gegenüber Migrantlnnen beeinflussen können. Sie betonen die Bedeutung von therapeuti- 
scher Bewegungsarbeit als sicheren Übergangsraum zwischen Vergangenheit und Zukunft für die Gegenwart und das Potential der „nonverbalen Integration ihrer Kultur durch gezielte Arbeit mit Körper und Bewegung“. Sie weisen auf die Dringlichkeit hin, Lösungen für inre Integration in die Aufnahmegesellschaften zu finden.

Dietrich-Hartwell, Goodill und Koch (2020) formulieren einen Leitfaden zur Tanz-, Bewegungstherapie im klinischen Setting einer traumatisch informierten, somatischen und psychologischen Versorgung von Flüchtlingen. Der Leitfaden gibt klinische Empfehlungen und beschreibt Herausforderungen und theoretische Überlegungen für die tanz-, bewegungstherapeutische Arbeit mit Flüchtlingen. Als Stundenstruktur und Techniken werden Einchecken, Aufwärmen, Themenentwicklung und Abkühlen empfohlen sowie rituelle Bewegung zu Beginn und am Ende unter Verwendung von Musik aus der Kultur. Besonders hervorgehoben wird die therapeutische Trauma-Haltung, die eine wichtige Voraussetzung zur Gewährleistung von Sicherheit, emotionaler Regulierung und Auffangen sei. Parallel organisierende Kinderbetreuung und Bereitstellung von Essen werden als gewinnbringende ergänzende Maßnahmen beschrieben. Als wirksames therapeutisches Element wird die Förderung des Verbundenheitsgefühls hervorgehoben, das für Flüchtlinge zentral sei, welches durch den / die Therapeutln und die Gruppenmitglieder mithilfe von spiegelnden Bewegungen, dem Zuhören von persönlichen Geschichten und dem Anschauen von Bildern ihrer Lieben gefördert werden könne.

Weidinger-von der Recke (2021) beschreibt in ihrer Fallvorstellung die Kombination von Einzel- und Gruppentherapie als notwendiges und sinnstiftendes Angebot zur Verarbeitung von Sozialem Trauma, mit Tanz-, Bewegungstherapie als festen Bestandteil und als Ergänzung zur tiefenpsychologisch fundierten Psychotherapie. Unter Sozialem Trauma werden
Ereignisse verstanden, „die einer spezifischen Gruppe von Menschen, also einer Gruppe sich bewegender fühlender Körper, angetan wurde“ (S.6). Diese Ereignisse sollen erfasst und verstanden werden.

Wichtig für den Beziehungsaufbau und den therapeutischen Erfolg sei die Orientierung an kulturellen und individuellen Ressourcen der Klientinnen, verbindliche Gruppenregeln, achtsame Kommunikation, Anwendung einfacher Bewegungs- und Wahrnehmungsangebote und das Hinzuziehen einer Sprach- und Kulturvermittlerin. In Bezug auf die Therapeutin sei die Anteilnahme und wohlwollende Neugier auf die fremde Kultur, Sprache und nonverbale Kommunikation für die Beziehung und den Erfolg ausschlaggebend.

\section{Schlussfolgerung}

Die Beiträge beschreiben für die tanz-, bewegungstherapeutische Arbeit mit Traumatisierten die Bedeutung der Einhaltung wiederkehrender Strukturen (Anfangs- und Endrituale, Einchecken, Aufwärmen, Themenentwicklung und Debriefing) und das Einbeziehen kulturell vertrauter Elemente und Ressourcen. Um Menschen anderer Kulturen respektvoll begegnen und sie adäquat therapeutisch fördern zu können, bedarf es auf Seiten der TherapeutInnen eine selbstreflektierende Haltung, Offenheit und Kultursensibilität.

\section{Literatur}

Aranda, E., Hills de Zárate, M., Panhofer, H. (2020): Transformed ground, transformed body: clinical implications for dance movement therapy with forced migrants. Body, Movement and Dance in Psychotherapy 15 (3), 156-170, https://doi.org/10.1080/17432979.2020.1779817 Chakraborty, S. (2020): Featured counter-trafficking program: Kolkata Sanved's model Sampoornata. Child Abuse \& Neglect 100, 104169, https://doi.org/10.1016/j.chiabu.2019.104169 
Dieterich-Hartwell, R., Goodill, S., Koch, S. (2020): Dance / movement therapy with resettled refugees: a guideline and framework based on empirical data. The Arts in Psychotherapy 69, 101664, https://doi.org/10.1016/j.aip.2020.101664 Grasser, L. R., Al-Saghir, H., Wanna, C., Spinei, J., Javanbakht, A. (2019): Moving through the trauma: dance / movement therapy as a somatic-based intervention for addressing trauma and stress among Syrian refugee children. Journal of the American Academy of Child and Adolescent Psychiatry 58 (11), 1124-1126, https:// doi.org/10.1016/j.jaac.2019.07.007

Weidinger-von der Recke, B. (2021): Dance / movement therapy and social trauma. In: Hamburger, A., Hancheva, C., Volkan, V.D. (Hrsg.): Social trauma - an interdisciplinary textbook. Springer, Cham, 123-129, https://doi.org/10.1007/978-3-030-47817-9_13

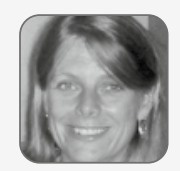

Dr. Iris Bräuninger Senior Researcher \& Co-Leiterin Studiengang Psychomotoriktherapie (Hochschule für Heilpädagogik Zürich IVE), Dozentin MA Tanztherapie UAB Barcelona, BTD-Supervisorin / Ausbilderin / Lehrtherapeutin, KMP-Notatorin, Praxis Tanztherapie Supervision Bodensee.

EDr. Iris Bräuninger dancetherapy@mac.com und iris.braeuninger@hfh.ch 